\title{
Unresectable Desmoid-Type Fibromatosis
}

National Cancer Institute

\section{Source}

National Cancer Institute. Unresectable Desmoid-Type Fibromatosis. NCI Thesaurus.

Code C155877.

Desmoid-type fibromatosis that is not amenable to surgical resection. 